\title{
Adolescent Burmese Refugee Perspectives on Determinants of Health
}

\author{
Avika Dixit, Emily M. Miner, Sarah E. Wiehe, Megan S. McHenry
}

\section{$\underline{\text { Abstract }}$}

Background

Over 70,000 Burmese refugees have resettled in the United States in the past decade.

While Burmese adolescents quickly acculturate into American society, their perspectives

on health are not well-known. The purpose of this study was to identify adolescent

Burmese refugee perspectives on determinants of health and health-related experiences

after resettlement.

Methods

In this qualitative study, Burmese adolescents took photographs depicting health-related experiences that were used as elicitation tools during focus groups. These discussions were recorded, transcribed, and analyzed for themes.

Results

Participants described positive determinants of health, including family and church.

Rampant tobacco use was identified by the participants as a determinant of poor health

within the Burmese community. Notably, the participants were proud to serve as liaisons within their community, despite the stressful nature of this role.

Discussion

Our results highlight the need to screen this population for anxiety, secondary to serving as a liaison for their community, as well as tobacco use.

Keywords: Adolescents, Refugees, Determinants of Health, Qualitative Research,

Burmese

This is the author's manuscript of the article published in final edited form as:

Dixit, A., Miner, E. M., Wiehe, S. E., \& McHenry, M. S. (2018). Adolescent Burmese Refugees Perspectives on Determinants of Health. Journal of Immigrant and Minority Health, 20(2), 370-379.

https://doi.org/10.1007/s10903-017-0648-7 


\section{Background}

Between 2005-2014, approximately 73,000 refugees from Burma were resettled in the United States (US) [1], Indianapolis is home to more than 12,000 Burmese refugees, of whom $86 \%$ are of Chin ethnicity [2]. The Chin State is located within the mountainous region of Myanmar, and they commonly have small farms in their villages[3].

Approximately $85 \%$ of the Chin refugees are Christians $[3,4]$. The Chin receive support not only from their family members but also their clan members. [3]. Among the Chin in Burma, practice of traditional medicine is uncommon in urban areas but may be seen in rural areas [3].

Nearly $40 \%$ of refugees are estimated to be under 18 years old [5]. Adolescent refugees face unique stressors during the resettlement process. After losing much of their social support, due to displacement from their homes and communities, separation from family members, disruption of school, they must assimilate into a new education system and learn a new language, with the potential for social exclusion, discrimination, and family conflict [6-8]. Few studies focus on healthcare needs specific to adolescent refugees internationally; mental health is typically the focus of these studies $[9,10]$.

Refugees have high rates of mental health disorders, with an estimated 3-30\% of adolescents suffering from depression, with pre- and post-migration adverse experiences as significant contributing factors [11]. A recent study found that Burmese adolescents served as a liaison for their parents and local community members, translating during many aspects of their daily lives, which is perceived to place considerable psychological 
burden on them [12]. The stress that this role may create is just one example of determinants of health within this population. Determinants of health are factors that affect health of individuals and communities and include: social and economic environment; physical environment; and individual characteristics and behaviors [13]. To our knowledge, there are no studies looking at refugee adolescent perspectives on determinants of health, which may provide understanding into the stressors experienced as refugees, as well as their general understanding of health.

Understanding individuals' perceptions and behaviors with respect to health can help inform health education programs and preventative services [14]. Programs and services customized to patient-specific needs have the ability to motivate changes in health-related behaviors $[15,16]$. Individuals, especially adolescents, are more likely to process medical information in a meaningful way when it is perceived to be relevant within the context of their lives and communities [15]. This, in turn, allows for opportunities to improve healthcare for vulnerable populations. Our objective in this study was to identify Burmese adolescent refugees’ perspectives on determinants of health and health-related experiences after resettlement in the US.

\section{Conceptual Framework}

With this study, we aimed to help healthcare providers understand the needs of these patients and reduce disparities in this population. We worked within Kilbourne et. al.'s conceptual framework on health disparities research agenda, with our study focused on “The Understanding Phase” (Figure 1) [14]. By identifying perceived determinants of 
health at the individual level, we aim to better understand health disparities in this population. In doing this, we hypothesized that we would gain better conceptualization of risk factors for mental health conditions in Burmese refugee adolescents. Our hypothesis is supported by findings from a recent study of African asylum-seekers and refugees in Hong Kong, which also utilized a social determinants of health framework to understand effects on mental health[17]. 


\begin{tabular}{|l|l|l|l|}
\hline Detecting \\
Define health disparities \\
Define vulnerable \\
populations \\
$\begin{array}{l}\text { Measure disparities in } \\
\text { vulnerable populations } \\
\text { Consider selection effects } \\
\text { and confounding factors }\end{array}$
\end{tabular}




\section{Methods}

\section{Participants}

The study was conducted in partnership with a local community organization that serves Burmese refugees. As per the suggestion of organizational leadership, we recruited from their summer college preparatory program for Burmese high school students, inviting all 27 students enrolled in summer 2014 to participate. Interested students were invited to submit a written application to be considered for participation, and selections were made after discussion between the program leadership and the authors (AD and MM). Selection criteria included having an interest in the project, availability of transport, and English proficiency in an attempt to select adolescents who were acculturated.

\section{Data Collection}

This cross-sectional study utilized photo-elicitation during focus groups (FGs). All participants (and parents/guardians for those $<18$ years) provided written informed assents and consents. Gift cards (\$40) and study cameras were used as incentives. Institutional Review Board approval was obtained prior to study initiation.

An initial session about photography, ethics, and safety was conducted by MM, AD, and a professional photographer. Participants’ photographs were used as elicitation tools to facilitate discussion among the participants during five FGs, conducted in English, from October 2014 to January 2015. Participants were given general broad topics to explore by taking photographs. The topics for first two focus groups were “determinants of good health and determinants of poor health.” Topics for the subsequent three photography 
assignments and focus groups were chosen based on the prominent themes of topics discussed during the two initial sessions, which included: relationships, community, traditions, and the adolescents' roles in their community. While not typical of photoelicitation methods such as photovoice, this study used initial themes to provide topics for subsequent discussion. The rationale for this was to provide some initial structure for participants within the broad constructs of social determinants of health, while allowing the participants to explore their own ideas in more depth during subsequent sessions. Participants selected and submitted three of their own photos for discussion prior to each focus group, regardless of whether they were in attendance. Discussions were moderated by the senior author (MM) who has extensive qualitative-experience in varied settings. General comments were invited for each photo. After an initial discussion, the participants who took the photos identified themselves and elaborated on their original interpretation of the image and further discussion followed. Each focus group lasted approximately 1-2 hours.

Photo-elicitation was chosen because it provides simple means to see the viewpoint of vulnerable populations. It allows participants to bring ideas or stories from other community members and from varied social and behavioral settings [18]. FGs were complementary to the photography as this method allows the participants to identify what the photographs represent to them and allow for their own interpretation. Exhibitions of selected photographs were conducted at a local art gallery and public library at the end of the study, in order to garner community exposure to this population [19]. 


\section{Measures}

As is inherent in the qualitative nature of our study, no objective measures were obtained.

\section{Analysis}

FGs were recorded and transcribed for data analysis. Thematic analysis using a phenomenological approach [20] was conducted using Dedoose software by AD, EM and MM independently [21]. The phenomenological approach allows researchers to extract phenomenon or experiences that is shared between individuals [20]. This approach was used to understand the contexts and situations that influenced or affected the adolescents' lived experiences and perspectives on the determinants of health. An initial codebook was created prior to the analysis based on a priori themes of interest and themes which emerged from the authors’ prior work with this population [12]. Codes were added throughout data analysis by an iterative process as new themes emerged with reapplication to previously analyzed transcripts. Disagreements and discordant codes were resolved after discussion among the authors involved in coding (AD, EM and MM). Overarching themes were identified using constant comparison analysis by the authors (AD, EM, and MM).

\section{$\underline{\text { Results }}$}

Ten participants were selected from students who expressed interest in participating in this study. One participant withdrew prior to the first meeting due to transportation issues. Seven female and two male students, all in grades 10 through 12, participated in the study. All participants were planning on applying for college in the coming years. 
Participant attendance at each group ranged from seven (78\%) to nine (100\%). Major themes that emerged from the FGs are discussed below with exemplar quotes.

\section{Family and Relationships}

The concept of health itself was synonymous with taking care of each other. An 'alone' individual was considered unhealthy, and the importance of family was frequently discussed.

"Health is all about taking care of each other" Most adolescents lived in large family units. An overarching theme was that of feeling safe when surrounded by family (Figure 2).

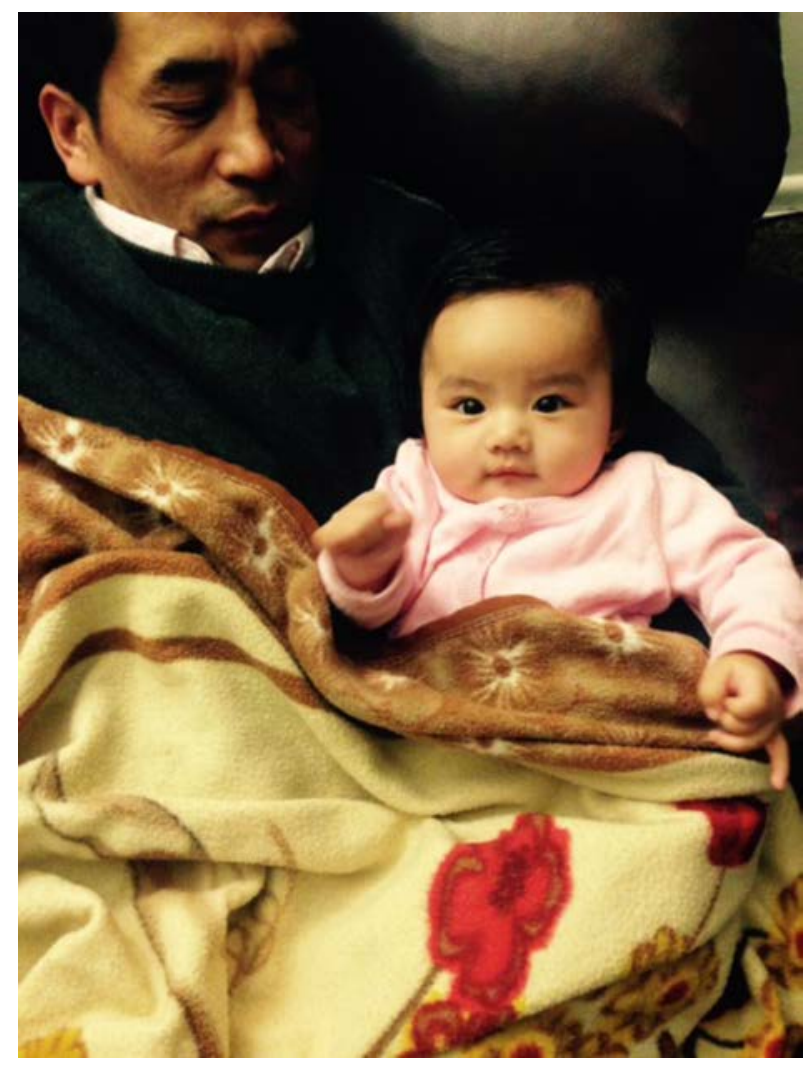


Figure 2: Photograph of a baby with grandfather that elicited a conversation around feeling safe with family: “The child looks like he is happy because he feels safe.”

The adolescents displayed a mature understanding of their parents' struggles throughout resettlement. Many showed respect to their parents by being obedient but experienced new conflicts with their parents after arriving in the US.

“After getting here everything has changed a lot. Back in Burma we don’t really talk back to our parents, but here, we talk back...because we see people here talk back to their parents.”

They noted that their parents felt alienated when they spent time alone in their room, compounded by the difference in skill level in speaking and understanding English.

"Mom really hates it when we're angry and we're alone. She feels like we don't trust her. And since she can't speak the language anymore, she's upset because she feels like we don't tell her much.”

Another source of conflict involved permission for the adolescents to date their peers, as most were not allowed to date. Arranged marriages were common in Burma, and a few adolescents’ older siblings had arranged marriages.

"If they talk to each other or people know they're dating, then their parents would get upset and they would look bad.”

However, since moving to the US, the adolescents believed their parents were open to letting them find their own partner.

"She said, as long as he loves me... then she's okay with it because she knows that I'm not going to be happy with an arranged marriage."

\section{Religion, Church and Community}


The freedom to practice their religion freely and without the fear of persecution after resettlement also emerged as a theme within these discussions. The adolescents recalled the fear of being shot if they freely displayed their religious beliefs prior to resettlement.

"We can't do [caroling] in Malaysia. We're going to be caught by bullets if we did it."

In their new communities, they described engaging in displays of religion, freely and without worry.

The adolescents indicated that church was a focal point of the Burmese community. Many church meetings were held at community members' houses, and the adolescents were friends with many of their churches' members. Adolescents recounted instances when a death in the family resulted in the church members mourning together and helping with funeral arrangements, expenses, and food.

"Chin people go to church, it’s very uncommon for people not to go to church.”

\section{Liaison Role}

The adolescents frequently served as liaisons for their community members. Examples of tasks they would perform included: translating bills and letters for their parents; tutoring for the state driving test; and driving people to buy groceries or to their appointments.

"I drive people everywhere...grocery, hospitals, church...”

They also commonly accompanied people to their doctors' appointments or the emergency room to help interpret information. The adolescents described performing these requests without protest. However, often, the adolescents were not allowed to interpret due to legalities or hospital policies, which was a source of frustration to them. 
The adolescents also expressed additional frustration about giving up their free time.

However, they expressed pride in helping their community.

“I think they're really proud of us for interpreting them. But sometimes they might feel sad because they can't communicate by themselves.”

Adolescents understood the importance of the tasks they were asked to do, and they often described the anxiety they felt when translating documents and phone conversations for their families. Bills were a particular source of stress for them (Figure 3).

"What if I translate it wrong? Or what if it's a totally different thing but I think it's this one. There's just a huge price to pay if I make one little mistake.”

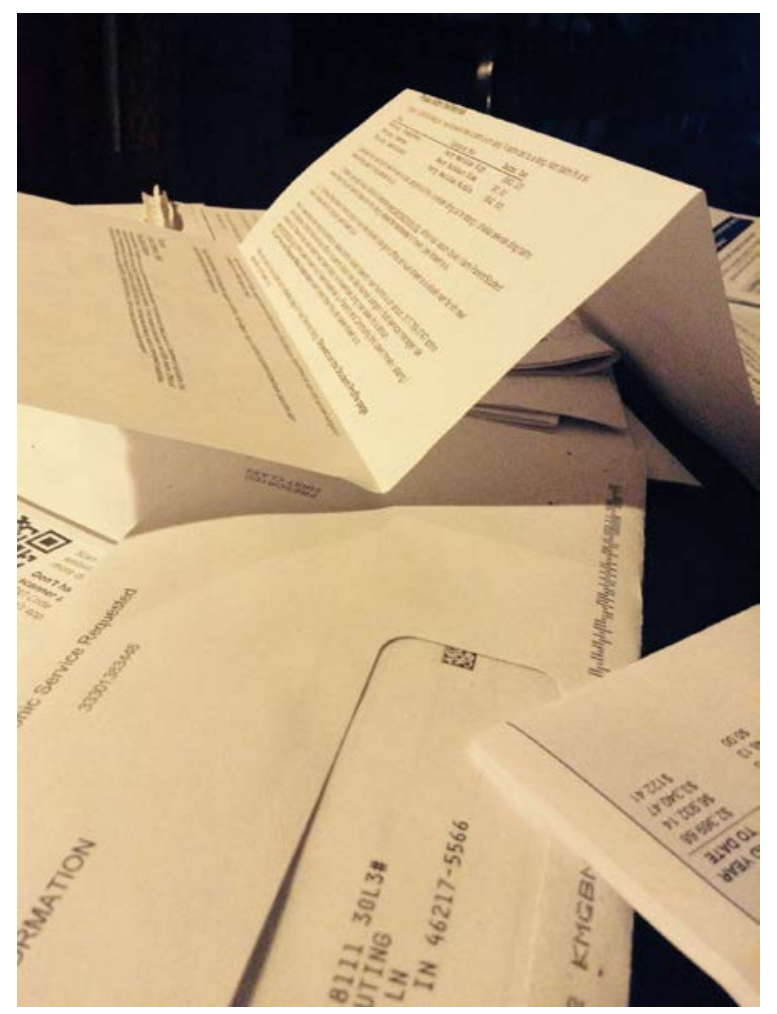

Figure 3: Picture showing bills and letters elicited a discussion of how adolescents were responsible for translating these documents for their families. 


\section{Food}

Great emphasis was placed on eating together as a family. The adolescents cherished these moments, even if their parents’ work schedules only allowed them to eat fast food together in a car. Adolescents described that cooking for their family was another way they showed respect and love.

"Dining together is good. But, most of our Chin community, our parents and our brothers and sisters, they work, so we don't get to dine together, so it's not healthy for our family."

Adolescents felt that fresh produce was easier to get in Burma and refugee camps. Many continued to grow their own food in the US.

“It’s free. It's fresh... We grow green peppers, basil, tomatoes... cabbage... chili peppers... eggplant... cucumbers.”

The adolescents considered processed or 'microwaveable' food as unhealthy, albeit convenient. The participants described major changes in their diet after resettlement. In addition to eating more convenience foods, they also ate more meat in the US, which was previously rarely available.

\section{Outdoor Environment}

Adolescents highlighted that being outside in nature had a positive impact on one's health. Standing water and trash were identified as concerning environmental issues within their community. Adolescents used photos of these items to emphasize their negative effects on their environments, such as a breeding ground for malaria-spreading mosquitos from their experience in refugee camps and poor sanitation.

\section{Physical Activity}


The participants frequently identified that physical activity leads to good health. While most of this discussion was around photos of individuals walking, running, or playing soccer outdoors, others added in photos of exercising indoors as another way to be active. While the participants agreed that physical activity was critically important, many admitted that they did not do it as much as they would like. A clear reason for this was not elucidated.

\section{Tobacco and Alcohol Use}

Many adolescents noticed high rates of tobacco and cigarette use in their community. They recognized cigarettes as being harmful and addictive (Figure 4).

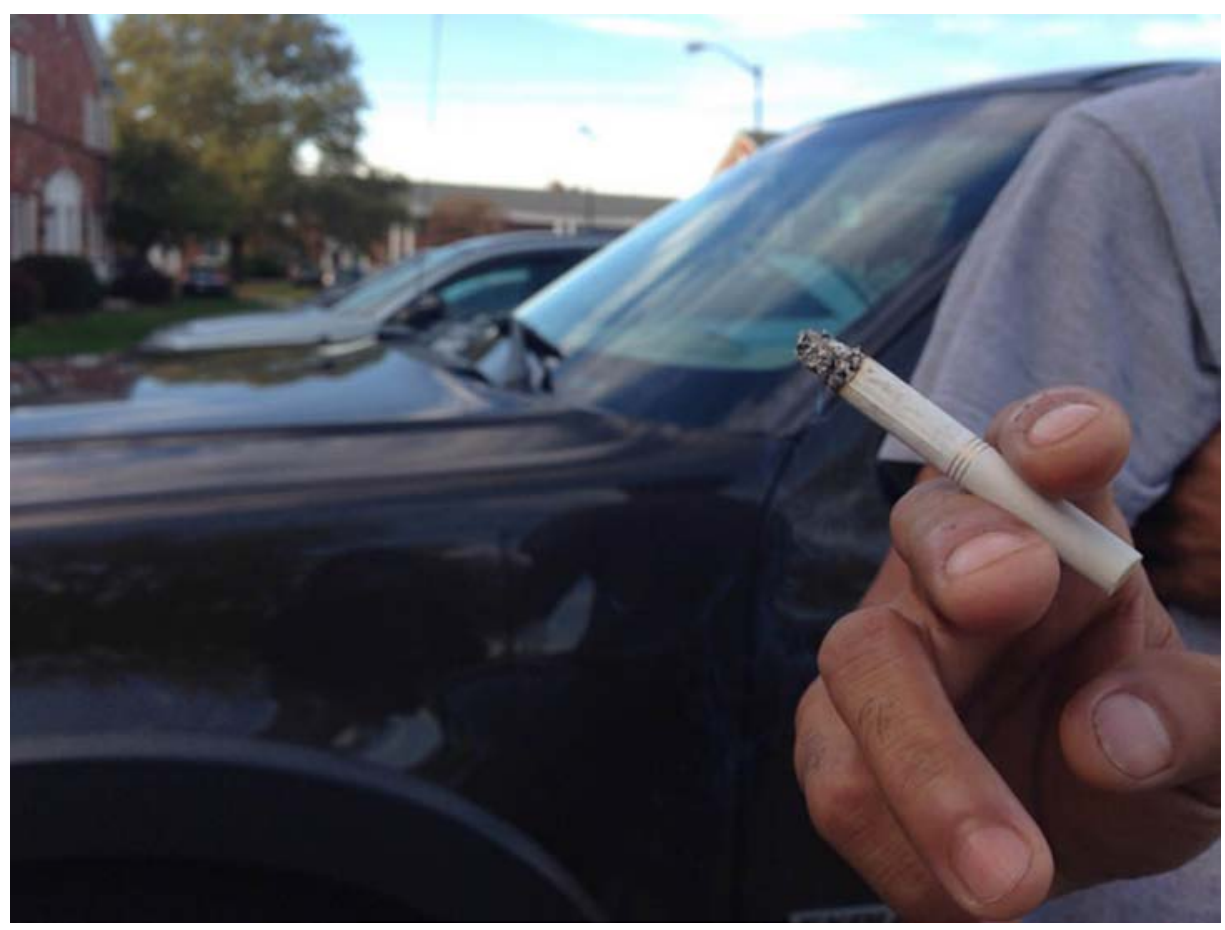

Figure 4: Photograph of a cigarette that led to a discussion about high consumption of tobacco and cigarettes in the Burmese community as perceived by the participants. 
They also highlighted the easy access that minors had to chewable tobacco at ethnic grocery stores where checking identification was uncommon.

"At the Asian grocery store...You don't have to have an ID...They use it kind of like candy.”

Alcohol use was identified as common in their community. Many adolescents noted harmful effects of alcohol dependence and abuse on health and relationships.

"Many people and teenagers in the Burmese community...they drink illegally or legally...drinking affects your life. And it wastes your money and your family. It damages everything."

Most adolescents reported that they do not have discussions with their parents about drugs or alcohol and that their parents rely on school to educate them about harmful effects of substance abuse.

\section{Technology}

Technology was also noted as detrimental to an individual's health. Although all of the adolescents admitted to frequently using technology, they perceived it as disruptive and a hindrance to family bonding.

"You touch your phone or whatever and, you're still talking, but you're not giving them your full attention. That's what is needed to build strong relationship."

This was another source of conflict with their parents.

"[Mother] wants to have a family together moment every day. You know? There's so many devices that we can touch, and we're all on our separate ways even if we're in the same room. We don't really communicate like we used to anymore because we're all on our own. And she really hates that. She wants us to communicate. She always tells us this, so we're making her mad." 


\section{Traditions and Culture}

The adolescents photographed traditional medicine practices, as they defined them: rolling a can with oil on someone's back to help with fever, pulling sections of hair for headaches, pricking finger tips to "let out bad blood" when ill. Participants felt that these were practiced due to lack of access to healthcare in Burma, and their continued use was only by older Burmese adults in the US.

There were multiple photos of young children and families, which were noted as an important part of the culture. During these discussions, infant feeding and sleep practices were highlighted. The adolescents noted that breastfeeding durations were overall longer for their community back in Burma compared to after resettlement in the US, due to acculturation and time constraints. Co-sleeping was considered a norm and a form of affection. Adolescents expressed surprise when they learned that most babies in the US do not sleep with their parents.

"Before we came here, I didn't even know that they let their kids, even toddlers, sleep alone. And I think that really affects the love between a child and a mother. Because in our culture they sleep with our parents until they're old enough to sleep on their own.”

\section{$\underline{\text { Discussion }}$}

Utilizing photo-elicitation, we sought to understand adolescent Burmese refugees’ perspectives on determinants of health. While we hypothesized that risk factors impacting mental health would be a focus within the group’s discussions, participants recognized a wide variety of determinants of health. Their discussions focused on themes which fell 
within all three categories defined by the World Health Organization: socio-economic, physical environment, and individual [13].

Being alone or separated from one's friends and family was considered unhealthy. This has vital implications for the adolescents' mental health as many have suffered separation from their family (e.g. one parent or family member being resettled prior to the rest of the family). Beyond the family unit, church was a supportive community for the participants. These findings are similar to those of a study that looked at young refugees resettled in Australia [22]. Family cohesion, faith, and religious involvement have been identified as protective factors against mental health disorders and promoters of psychosocial wellbeing among adolescent refugees [23,24]. Additionally, their freedom to practice their religion freely in the US was liberating to them.

The adolescents reported some conflicts with their parents, a developmentally expected phenomenon with acquisition of independence. For Burmese adolescents, this normal transition was compounded by faster assimilation as compared to their parents. Additionally, the omnipresence of technology, which adolescents generally use more than parents, further contributed to conflicts. This is similar to the parent-child communication barriers noted in other refugee and immigrant groups [6,25-27].

Our study confirmed that many Burmese refugee adolescents in Indianapolis serve as "culture brokers" (CB) for their families and their communities. Participants in our study reported a sense of pride in this role and such a role has been associated with a sense of 
efficacy in other immigrant adolescents [26]. This role has been noted to have both positive and negative effects [27]. Based on survey and observational data, sixth grade Latino children who identified themselves as translators for their families did significantly better on standardized tests than children who did not translate [28]. However, in another study of Latina adolescents, assuming such a role was associated with higher work and school stress, as well as lower academic achievement [29]. Similar negative mental and emotional reactions have been experiences by other ethnic groups, such as former Soviet Jewish families [30], Vietnamese families [25], and Cuban refugee families [31]. Providers should be aware of the burden this role can bear on adolescents and screen for mental health conditions, such as anxiety and depression, and intervene appropriately.

Despite the fact that Title VI of the Civil Rights Act of 1964 requires healthcare providers receiving federal funding to offer language assistance [32], some providers continue to use ad-hoc interpreters whose errors are more likely to have clinical consequence [33]. Many states and hospital systems prohibit such practices. However, because the restriction of ad-hoc interpreters is inconsistent, Burmese refugees continue to ask the English proficient teenagers within their community to accompany them to the clinic or hospital. Stories of conflicts and frustrations occurring within the clinical setting were common whenever they were unable to help translate for their families. Providers in these situations can help decrease the adolescents' frustrations by acknowledging their time and effort but explaining the reason why hospitals may refuse their assistance for interpreting. 
Participants described changes in their diet post-resettlement, with more reliance on processed and fast food, as unhealthy but convenient. Almost all participants identified physical activity as important but felt that they were not engaging in it as much as they would like. Higher risk for weight gain after resettlement has been identified in other pediatric immigrant and refugee populations [34-36]. Monitoring physical activity and body mass index changes is standard practice for most pediatric providers, but interventions are often ineffective due to the chronicity of obesity. Since weight gain in refugees occurs specifically after resettlement, counseling on culturally appropriate healthy eating and exercise may be more impactful in this population, especially if instituted at the time of resettlement.

The adolescents observed traditional health practices in their community, which were thought to be a temporizing measure until a healthcare provider could be seen. These can be classified into three categories [37]: (1) result in no harmful health effects (canrolling), (2) produce beneficial health outcomes (longer breastfeeding), and (3) have serious, harmful health outcomes (finger-pricking for blood-letting). The latter, fingerpricking, has been shown to increase the risk for transmission of blood-borne viruses similar to risk seen in unsafe blood glucose monitoring in patients with diabetes [38]. While it is beneficial for medical providers to be aware of this, the frequencies of these practices were reported by our participants to be decreasing. 
The adolescents noted that breastfeeding duration has decreased in their community after resettlement, similar to findings among more acculturated Hispanic immigrants [39]. A review of migrant and refugee women found that they face multiple challenges when attempting to breastfeed in a new country and require culturally appropriate support from healthcare providers [40]. This should be an important consideration for pediatric providers caring for young Burmese infants.

Based on the adolescents' perceptions, co-sleeping was considered normal and a form of affection within this Burmese community. Similar attitudes have been observed in other Asian cultures [41]. This differs from the American Academy of Pediatrics’ recommendations [42]. To ensure culturally appropriate care, healthcare providers should consider a harm-reduction approach when tailoring their advice about bed-sharing, rather than an automatic condemnation of co-sleeping [43].

Based on our findings, future research should focus on identifying gaps in public health programs and policy related to Burmese refugees. This may then inform best practices for practitioners caring for this population. Our study suggests that potential areas for further evaluation may include screening refugee adolescents for stress arising from their liaison role; supporting breastfeeding mothers; and culturally sensitive harm-reduction approaches when faced with co-sleeping. Additionally, emphasis should be placed on curbing access to tobacco products for minors through collaborative public health interventions aimed at education both teens and sellers of such products. 


\section{Limitations}

Although our sample size was small, it allowed for individualized training in photography and ethics for the participants. The participants were selected from those considering college, and all except two were females. Hence, these results may not be representative of all Burmese adolescent refugees. Our demographic data were limited in that we did not collect specific data on the number of years the participants lived in the US, their specific Burmese ethnicity, or their religion. We tried to mitigate these limitations by obtaining views of the broader community using photo-elicitation [18]. Use of FGs allowed for collection of data directly from participants, rather than the researchers' interpretation of the photographs.

\section{New Contribution to the Literature}

To our knowledge, this is the first study looking at perspectives of determinants of health from Burmese adolescent refugees. We found that Burmese adolescents face similar challenges as other immigrant and refugee adolescents in a liaison role and that this can be a sense of pride for the adolescent. Our study findings have important implications for providers caring for Burmese families, including effect of lifestyle changes on health, family relationships, and tobacco and alcohol use, as well as infant care practices. 


\section{Acknowledgements}

The authors would like to thank the adolescents who participated in this project. This study was funded by the Community Access to Child Health (CATCH) Resident Grant from the American Academy of Pediatrics. We are thankful for support from the Burmese American Community Institute (BACI), especially Elaisa Vahnie (Executive Director of BACI), Lian Sang (BACI Program Associate) and Lian Ceu (BACI Upward College Program Director). Thanks to Abbey Bullerdick and Abby Hacker for providing photography expertise. Thanks to the Harrison Center for the Arts and Indianapolis Public Library, Central Library for holding an exhibit of the photographs taken as a part of this project. We appreciate the support from the Morris Green Scholars Program, Indiana University School of Medicine. 


\section{References}

1. Tan V. US wraps up group resettlement for Myanmar refugees in Thailand [Internet]. UNHCR. 2014 [cited 2015 Jul 19]. Available from:

http://www.unhcr.org/52e90f8f6.html

2. Burmese American Community Institute. Burmese Refugee Population in the US BACI Official website [Internet]. Burmese Am. Community Inst. 2015 [cited 2015 Jul 19]. Available from: http://www.baci-indy.org/resources/burmese-refugee-population-inthe-us

3. Sandy Barron, John Okell, Saw Myat Yin, Kenneth VanBik, Arthur Swain, Emma Larkin, et al. Refugees from Burma: Their Backgrounds and Refugee Experiences. Washington, DC: Center for Applied Linguistics; 2007 Jun. Report No.: Cultural Profile No. 21.

4. The International Center. The Burmese Community in Indiana: Fact Sheet. The International Center; 2013.

5. United Nations Children's Fund. Uprooted: The growing crisis for refugee and migrant children [Internet]. New York, NY; 2016. Available from:

http://www.unicef.org/publications/index_92710.html

6. Meschke LL, Juang LP. Obstacles to parent-adolescent communication in Hmong American families: exploring pathways to adolescent mental health promotion. Ethn. Health. 2014;19:144-59.

7. Lustig SL, Kia-Keating M, Knight WG, Geltman P, Ellis H, Kinzie JD, et al. Review of child and adolescent refugee mental health. J. Am. Acad. Child Adolesc. Psychiatry. 2004;43:24-36.

8. Measham T, Guzder J, Rousseau C, Pacione L, Blais-McPherson M, Nadeau L. Refugee Children and Their Families: Supporting Psychological Well-Being and Positive Adaptation Following Migration. Curr. Probl. Pediatr. Adolesc. Health Care. 2014;44:208-15.

9. Elklit A, Østergård Kjær K, Lasgaard M, Palic S. Social support, coping and posttraumatic stress symptoms in young refugees. Torture. 2012;22:11-23.

10. Betancourt TS, Newnham EA, Layne CM, Kim S, Steinberg AM, Ellis H, et al. Trauma History and Psychopathology in War-Affected Refugee Children Referred for Trauma-Related Mental Health Services in the United States. J. Trauma. Stress. 2012;25:682-90.

11. Bronstein I, Montgomery P. Psychological Distress in Refugee Children: A Systematic Review. Clin. Child Fam. Psychol. Rev. 2011;14:44-56. 
12. McHenry M, Umoren R, Dixit A, Holliday R, Litzelman D. Exploring Healthcare Perspectives of Burmese Chin Refugees. J. Cult. Divers. 2016;23:151-7.

13. WHO. The determinants of health [Internet]. World Health Organ. [cited 2017 Feb 28]. Available from: http://www.who.int/hia/evidence/doh/en/

14. Kilbourne AM, Switzer G, Hyman K, Crowley-Matoka M, Fine MJ. Advancing Health Disparities Research Within the Health Care System: A Conceptual Framework. Am. J. Public Health. 2006;96:2113-21.

15. Krebs P, Prochaska JO, Rossi JS. A meta-analysis of computer-tailored interventions for health behavior change. Prev. Med. 2010;51:214-21.

16. Kreuter MW, Wray RJ. Tailored and targeted health communication: strategies for enhancing information relevance. Am. J. Health Behav. 2003;27 Suppl 3:S227-232.

17. Wong WCW, Cheung S, Miu HYH, Chen J, Loper KA, Holroyd E. Mental health of African asylum-seekers and refugees in Hong Kong: using the social determinants of health framework. BMC Public Health. 2017;17:153.

18. Wang C, Burris MA. Photovoice: Concept, Methodology, and Use for Participatory Needs Assessment. Health Educ. Behav. 1997;24:369-87.

19. ThemeFuse. The PhotoVoice Project | Harrison Center for the Arts [Internet]. [cited 2017 Feb 27]. Available from: http://harrisoncenter.org/the-photovoice-project/

20. Creswell JW, Creswell JW. Qualitative inquiry \& research design: choosing among five approaches. 2nd ed. Thousand Oaks: Sage Publications; 2007.

21. Dedoose: Web application for managing, analyzing, and presenting qualitative and mixed method research data [Internet]. Los Angeles, CA: SocioCultural Research Consultants, LLC; 2016. Available from: www.dedoose.com

22. Earnest J, Mansi R, Bayati S, Earnest JA, Thompson SC. Resettlement experiences and resilience in refugee youth in Perth, Western Australia. BMC Res. Notes [Internet]. 2015 [cited 2015 Oct 22];8. Available from: http://www.biomedcentral.com/17560500/8/236

23. Weine S, Ware N, Hakizimana L, Tugenberg T, Currie M, Dahnweih G, et al. Fostering Resilience: Protective Agents, Resources, and Mechanisms for Adolescent Refugees’ Psychosocial Well-Being. Adolesc. Psychiatry. 2014;4:164-76.

24. Fazel M, Reed RV, Panter-Brick C, Stein A. Mental health of displaced and refugee children resettled in high-income countries: risk and protective factors. The Lancet. 2012;379:266-282.

25. Trickett EJ, Jones CJ. Adolescent culture brokering and family functioning: A study of families from Vietnam. Cultur. Divers. Ethnic Minor. Psychol. 2007;13:143-50. 
26. Wu NH, Kim SY. Chinese American adolescents' perceptions of the language brokering experience as a sense of burden and sense of efficacy. J. Youth Adolesc. 2009;38:703-18.

27. Jones CJ, Trickett EJ, Birman D. Determinants and consequences of child culture brokering in families from the former Soviet Union. Am. J. Community Psychol. 2012;50:182-96.

28. Orellana MF. Responsibilities of children in Latino immigrant homes. New Dir. Youth Dev. 2003;25-39.

29. Sy SR. Family and Work Influences on the Transition to College Among Latina Adolescents. Hisp. J. Behav. Sci. 2006;28:368-86.

30. Jones CJ, Trickett EJ. Immigrant adolescents behaving as culture brokers: a study of families from the former Soviet Union. J. Soc. Psychol. 2005;145:405-27.

31. Puig ME. The Adultification of Refugee Children: Implications for Cross-Cultural Social Work Practice. J. Hum. Behav. Soc. Environ. 2002;5:85-95.

32. Chen AH, Youdelman MK, Brooks J. The Legal Framework for Language Access in Healthcare Settings: Title VI and Beyond. J. Gen. Intern. Med. 2007;22:362-7.

33. Flores G, Laws MB, Mayo SJ, Zuckerman B, Abreu M, Medina L, et al. Errors in medical interpretation and their potential clinical consequences in pediatric encounters. Pediatrics. 2003;111:6-14.

34. Heney JH, Dimock CC, Friedman JF, Lewis C. Pediatric refugees in Rhode Island: increases in BMI percentile, overweight, and obesity following resettlement. R. I. Med. J. 2013. 2015;98:43-7.

35. Hervey K, Vargas D, Klesges L, Fischer PR, Trippel S, Juhn YJ. Overweight among Refugee Children after Arrival in the United States. J. Health Care Poor Underserved. 2009;20:246-56.

36. Magnusson MB, Hulthén L, Kjellgren KI. Obesity, dietary pattern and physical activity among children in a suburb with a high proportion of immigrants. J. Hum. Nutr. Diet. 2005;18:187-94.

37. Morisky DE. Traditional Health Beliefs, Practices. [Internet]. encyclopedia.com. 2002 [cited 2015 Aug 2]. Available from: http://www.encyclopedia.com/doc/1G23404000860.html

38. Klonoff DC, Perz JF. Assisted Monitoring of Blood Glucose: Special Safety Needs for a New Paradigm in Testing Glucose. J. Diabetes Sci. Technol. 2010;4:1027-31. 
39. Singh GK, Kogan MD, Dee DL. Nativity/Immigrant Status, Race/Ethnicity, and Socioeconomic Determinants of Breastfeeding Initiation and Duration in the United States, 2003. Pediatrics. 2007;119:S38-46.

40. Schmied V, Olley H, Burns E, Duff M, Dennis C-L, Dahlen HG. Contradictions and conflict: A meta-ethnographic study of migrant women's experiences of breastfeeding in a new country. BMC Pregnancy Childbirth. 2012;12:163.

41. Dwivedi KN. Meeting the Needs of Ethnic Minority Children: Including Refugee, Black, and Mixed Parentage Children : a Handbook for Professionals. Jessica Kingsley Publishers; 2002.

42. Task Force on Sudden Infant Death Syndrome. SIDS and Other Sleep-Related Infant Deaths: Expansion of Recommendations for a Safe Infant Sleeping Environment.

Pediatrics. 2011;128:1030-9.

43. Ward TCS, Doering JJ. Application of a Socio-Ecological Model to Mother-Infant Bed-Sharing. Health Educ. Behav. 2014;41:577-89. 
Appendix I: Interview Guide for Focus Group Discussions

[Original template is provided here. This was slightly altered such that the entire group discussed the photograph prior to the explanation from the participant who took the photograph.]

Facilitator's Name: Date:

Observer/Notetaker’s Name:

Topic of discussion (assigned at last meeting):

Other study team members present:

Hello and welcome to this group discussion. My name is and I will help guide our discussion today. is also present today as an observer to help record our conversations. This helps us to capture what you say, not what we think you said. We will go around the table and discuss your selected photographs for the topic assigned at our last meeting.

In this group, we consider you the teachers. We want your honest opinions and ideas. We want to be very clear that anything said in this group discussion will be kept private and confidential. We will not be sharing your answers with anyone, and the recordings of this conversation will not have your names attached to it. You should feel free to say whatever you think and feel. You are welcome to say as much or as little as you want. We are eager to hear your words and to hear what you have to say. If you are uncomfortable with a question, you can decide not to answer it. If this happens, it would be useful to us if you can tell us why you are uncomfortable with a particular question. But if that would also make you feel uncomfortable, then you do not have to say anything. Does anyone have any questions about what I have said so far?

\section{Ground rules}

Before we begin, I would like for us to all agree on how this discussion will proceed. First, if you have a mobile phone, please turn it off or turn it to silent. Next, please speak loudly. We are tape recording this conversation, and your opinions are VERY important to us. We want to be sure to be accurate in what you say. Recording this group is the best way for us to focus on what we are talking about so we don't have to try and take notes. To help us, speak loudly and clearly. Third, please do not talk over each other. I am sure we have some strong opinions, and that is good. We want to hear your thoughts! But it is very difficult to capture the voices of more than one person on the tape recorder if two people are speaking at once. Also, by speaking one at a time, it will allow all of our sisters and brothers an opportunity to speak and contribute to the discussion. Finally, understand that even if we disagree with one another, all opinions are important. There are no right or wrong answers to these questions, only very important information that you can provide to help. Are there any questions? May we begin? 


\section{1) Opening:}

a) Please tell the group the name that you are called by. We will go around the circle and hear from everyone.

Now let us start at one end of the table. Each participant can either show and/or pass around the photo that we are discussing.

\section{2) Clarifying Questions (for each participant and photo)}

a) Briefly describe this photo that you have selected.

b) Why did you choose to share this specific photograph?

c) What is the real story that this photo tells?

d) Please tell me more about what's going on in this picture [may want to point out a particular activity or relationship in the photo].

e) Please tell me more about the people in this picture. Why did you want to focus on them?

f) Tell me more about why you took this picture.

\section{3) Probing Questions}

a) I see that in this photo, you highlight . Tell me more about this and how it [affects your health; affects the health of your community].

b) If you had taken a similar picture of these people/this activity, say, 5 years before, would the picture show something different?

c) What does this photo not show?

d) How is this photo different than the others you took for this topic? How is it similar? 
Appendix II: Additional photographs submitted by the participants

Theme: Tobacco

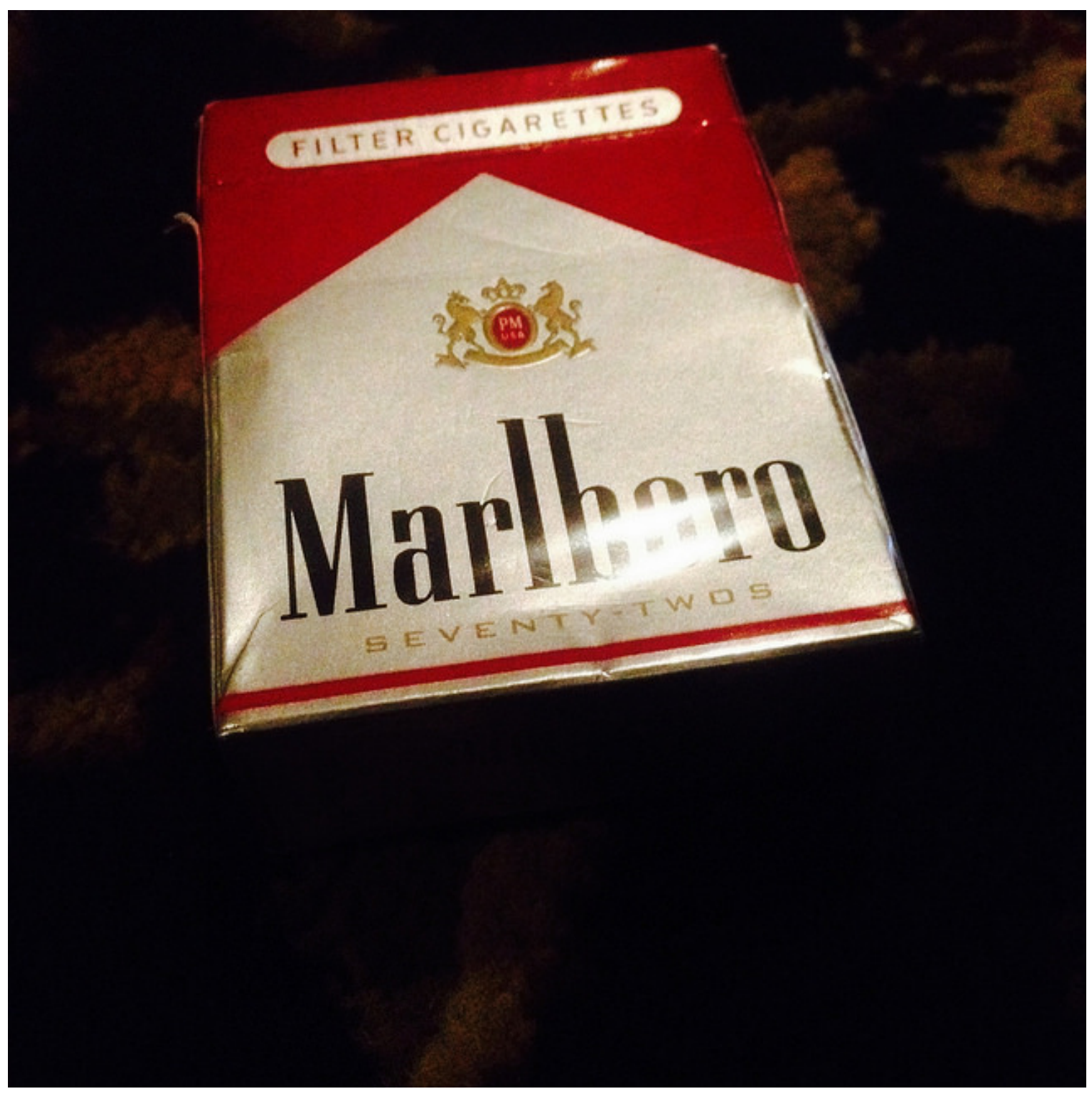

Figure A: Photo of a cigarette box elicited discussion about harmful effect of smoking on health 
Theme: Technology

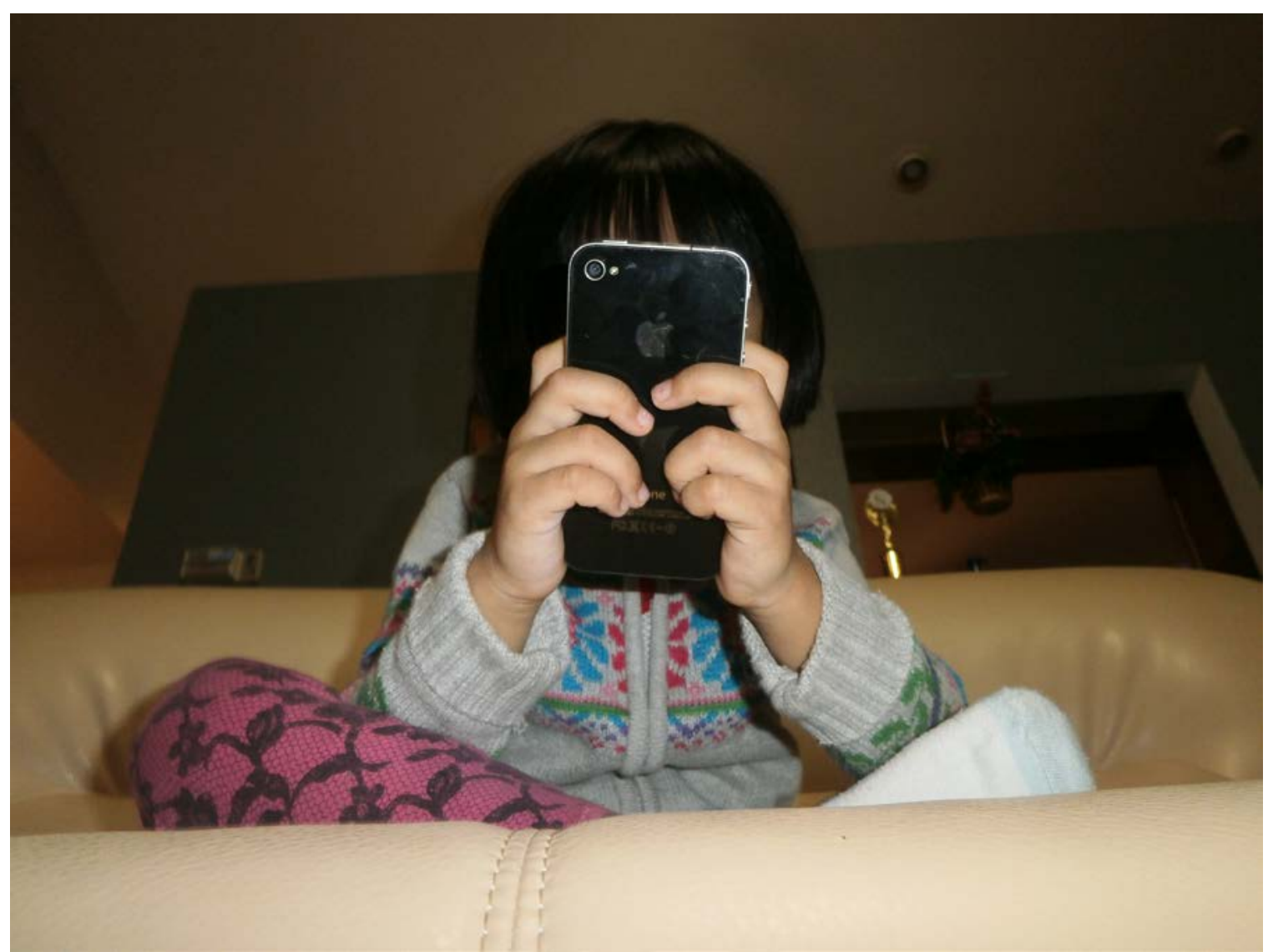

Figure B: Photo of a participant's sibling using a smartphone elicited discussion around technology's impact in the adolescents' lives 
Theme: Physical Activity

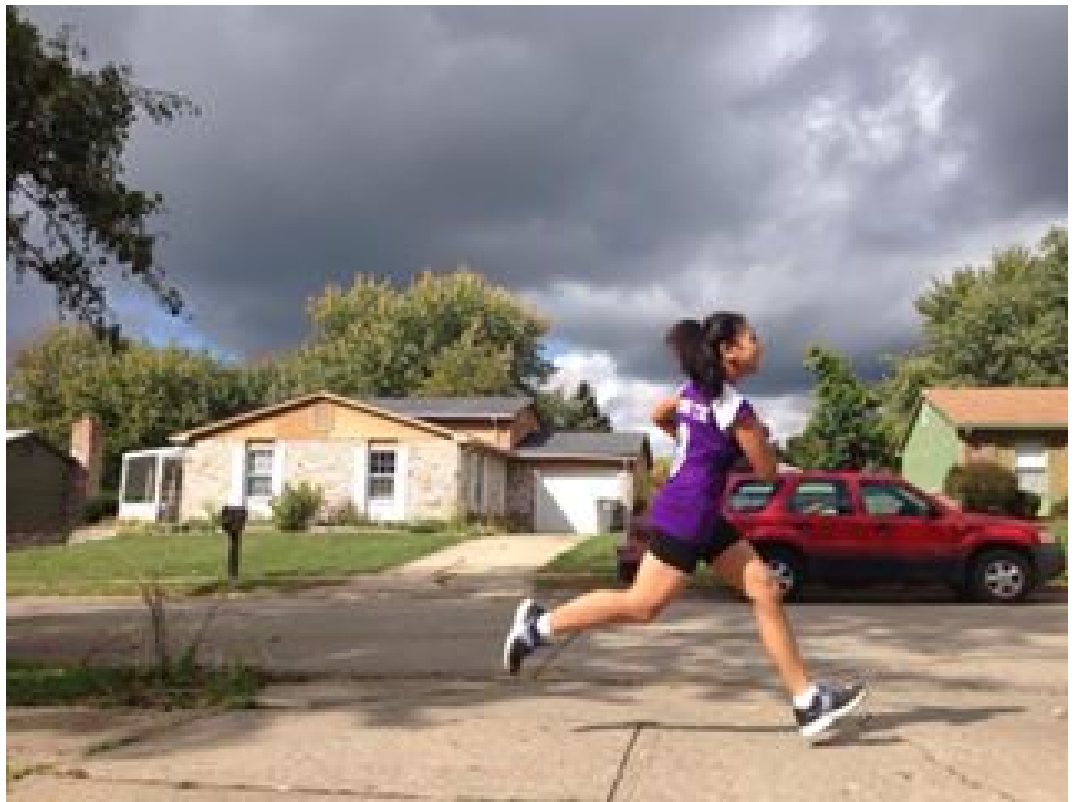

Figure C: Photo of a participants' sister running elicited discussion about physical activity as a positive influence on health 